\title{
Pathological remodelling of the athlete's heart
}

\author{
Tracey Keteepe-Arachi, Sanjay Sharma
}

St George's University Hospital, University of London, United Kingdom

\section{Summary}

Engaging in regular physical activity affords significant health benefits, while a lack of exercise is associated with increased cardiovascular morbidity and mortality. The amount of physical activity required to achieve these benefits is relatively modest, equating to 20-30 minutes of moderate intensity exercise per day. Most athletes far exceed these recommendations and now perform athletic feats previously considered insurmountable. Studies in veteran athletes have demonstrated favourable outcomes such as reduced incidence of cardiac disease and a greater lifespan. However, in asymptomatic athletes concealing underlying cardiac disease, intense exercise may be associated with sudden cardiac death. Despite the devastating and highly visible effects of life-threatening arrhythmias, exercise has retained its reputation as a prolonger of life as it is not the primary cause of death but merely a trigger for such catastrophes. More recent studies, however, have suggested that the effects of regular intense exercise may result in adverse remodelling in otherwise healthy hearts. This article will explore this concept and whether chronic intensive exercise can adversely impact cardiovascular health.

Key words: athlete's heart; adverse cardiac remodelling; atrial fibrillation; sudden cardiac death; veteran athlete; sports cardiology

\section{Introduction}

The World Health Organisation estimates physical inactivity causes 3.2 million deaths a year worldwide, despite the well-documented and indisputable benefits of exercise. In the USA, the mass uptake of exercise in the late 70 's resulted in a $48 \%$ reduction in heart disease over the subsequent two decades [1]. Regardless, there has been a resurgence of obesity in the West, and in the United Kingdom more than $60 \%$ of adults do not meet the government's recommendations for physical activity [2]. However, those who participate in regular exercise benefit from an advantageous cardiovascular risk profile, reduced incidence of heart disease, thus halving their risk of myocardial infarction. Dubbed the cheapest, most effective therapy a physician can pre- scribe, exercise is considered a holistic remedy, antidepressant and an extender of life.

While these benefits are the product of moderate physical activity, athletes far surpass the recommended duration and intensity of exercise. The cardiovascular modifications that occur as a result of the increase in cardiac output required for intense, prolonged exercise results in a pattern of structural and functional cardiac adaptations such as chamber dilatation, ventricular hypertrophy, enhanced diastolic filling and changes in autonomic function [3] (fig. 1).

A small proportion of athletes may occasionally demonstrate striking electrical and structural modifications that overlap with those observed in cardiac conditions implicated in exercise-related sudden cardiac death (SCD). Thus differentiating physiology from cardiac pathology in such cases can prove a challenging diagnostic conundrum. Alongside these findings, there are emerging data suggesting that chronic vigorous exercise can result in detrimental structural and electrical remodelling in normal hearts.

\section{Benefits of exercise}

More than 60 years ago a seminal study by Morris et al. marked the beginning of a barrage of studies documenting the benefits of exercise. The research documented a 50\% reduction in coronary artery disease in London bus conductors compared with their sedentary driver colleagues [4]. Since then it has been well documented that individuals who exercise regularly develop a favourable cardiovascular risk profile. Specifically, physical activity has been shown to improve blood pressure control, lipid profile and increase insulin sensitivity [5-7]. The benefits are observed not only in primary risk reduction but also in secondary prevention, and evidence demonstrating the benefits of exercise is well established in cardiac rehabilitation [8]. Heart failure patients who engage in physical activity demonstrate improvements in quality of life and functional capacity as well as a reduction in hospitalisations related to heart failure [9]. A meta-analysis of 34 


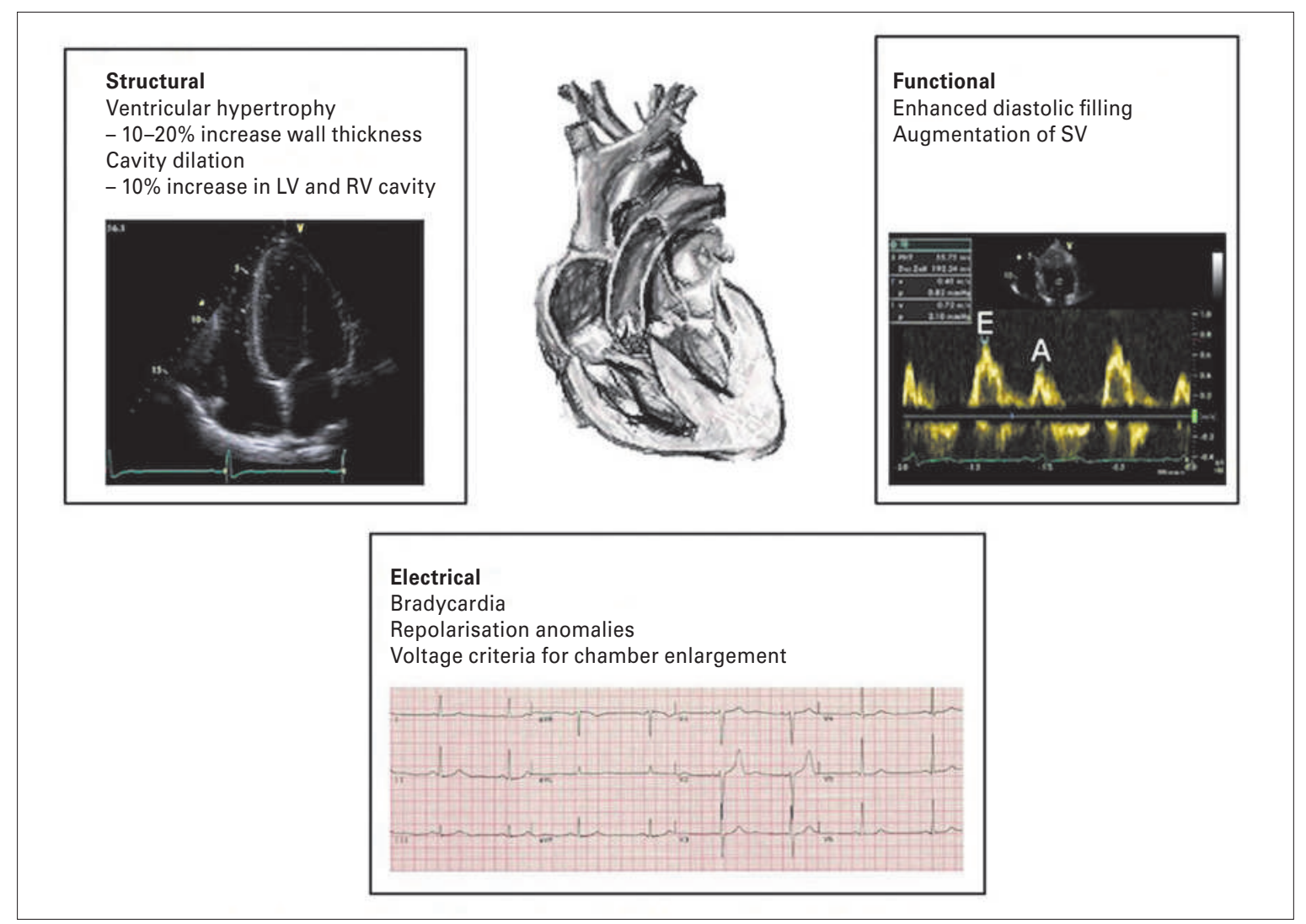

Figure 1: Structural, functional and electrical adaptations in the athlete's heart.

$\mathrm{LV}=$ left ventricle; $\mathrm{RV}=$ right ventricle; $\mathrm{SV}=$ stroke volume.

randomised controlled trials evaluating the effectiveness of exercise in cardiac rehabilitation after myocardial infarction showed a lower rate of reinfarction, and cardiac and all-cause mortality in the exercise-based cardiac rehabilitation group [10].

The mechanisms underlying these benefits are thought to be linked to modulation of signalling pathways influencing cardiac remodelling as well as the favourable effects of exercise in modifying other risk factors contributing to coronary artery disease. Physical activity has also been implicated in a lower prevalence of atrial fibrillation (AF) in individuals of increasing age, likely secondary to the beneficial effects of physical activity on other risk factors for AF such as ischaemic heart disease and hypertension [11].

Unsurprisingly, increased life expectancy in athletes compared with sedentary individuals has been convincingly demonstrated time and again. In a study of 15000 Olympic athletes, 3 years was added to their life-expectancy compared with those who did not exercise at all [12]. In fact a longevity benefit of up to 6 years was demonstrated in a study of 2600 Finnish elite athletes when compared with army recruits [13].
There may be other factors influencing this survival benefit, such as a healthier lifestyle in those who exercise regularly or genetic differences enabling more intensive exercise.

However, exercise not only provides cardiovascular protection but can also prevent osteoporosis, and reduce rates of depression and certain cancers including breast and prostate [14, 15] (fig. 2).

\section{Current exercise recommendations}

The recommended dose of physical activity for healthy adults from the British Association of Sports and Exercise Sciences and the US Department of Health and Human Services is 150 minutes of moderate-intensity physical activity or at least 75 minutes of vigorousintensity exercise per week [16]. Activity causing an increased pulse and raised respiratory rate but with preserved ability to speak is deemed moderate intensity. It is evident that regular physical activity is necessary for the reduction of chronic disease and prevention of premature death. However, only a modest amount of exercise is required to yield such benefits. 


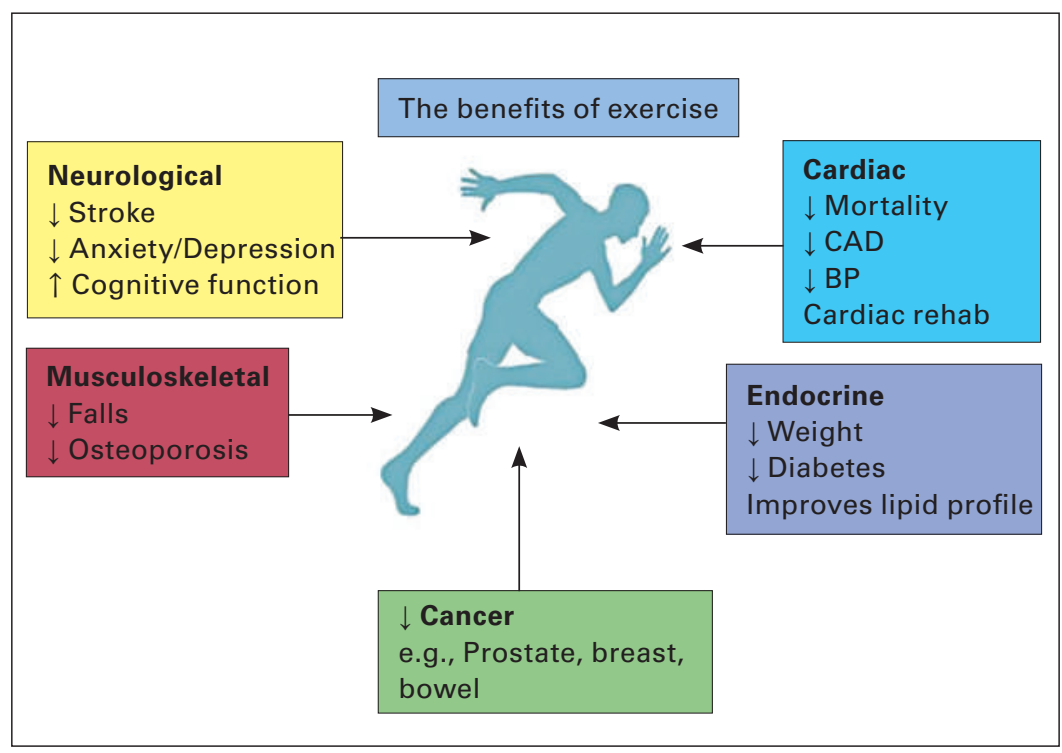

Figure 2: The benefits of exercise.

$\mathrm{CAD}=$ coronary artery disease $\mathrm{BP}=$ blood pressure.

For example, cycling at a pace of $15-20 \mathrm{~km} /$ hour, jogging at a pace of $6.4-8 \mathrm{~km} /$ hour or even a brisk walk constitutes activity equating to an intensity of 6-8 metabolic equivalent of tasks (METS) [17, 18].

\section{Sudden cardiac death related to sport}

Rarely, a young athlete may die suddenly during competition or immediately afterwards. Such catastrophes are uncommon with the prevalence ranging from 1 in 50000 to $1 \mathrm{in} 200000$ depending on the age of the athlete $[19,20]$. However, they are made highly visible through extensive media coverage, by virtue of the athlete's role model status and the common understanding that they are the healthiest and fittest members of society. In young athletes, below the age of 35 years, SCD is generally caused by genetic or congenital abnormalities affecting heart muscle, coronary arteries and the electrical system. For example, the cardiomyopathies are the commonest inherited cause of SCD whereas anomalous coronary arteries are the commonest congenital cause. Individuals harbouring these diseases increase their risk of SCD three-fold.

In addition to the pathological substrate, the neurohormonal, mechanical and metabolic stress of exercise serves as a trigger for fatal arrhythmias. Deaths are significantly more common in males, with a 9:1 male to female ratio in young athletes and a 20:1 ratio in recreational athletes. In reality most deaths in sport are in middle-aged recreational athletes and are generally attributable to atherosclerotic coronary artery disease (CAD) [21, 22]. These individuals usually have pre-existing risk factors for $\mathrm{CAD}$ although presence of disease is rarely revealed on the surface ECG. Consequently, the reputation of exercise remains intact due to its role as a trigger rather than a direct cause of SCD.

\section{Is there a deleterious effect of too much sport?}

The ultimate question generating considerable scientific interest is whether there is a direct dose-benefit relationship between exercise and cardiac health, or whether there is a dose beyond which there is a diminished return and perhaps a deleterious effect. The issue is pertinent because endurance events have become popular over the past two decades and there are an increasing number of individuals engaging in multiple sporting activities every year, such as the marathon and iron man. Furthermore, elite endurance athletes exercise at workloads 5-10 times greater than current recommended limits.

In a study of over 5000 male veteran athletes, a 13\% reduction in all-cause cardiovascular mortality was demonstrated for every MET of exercise performed above 4 METS, although the benefit did not extend beyond 10 METS [23]. A study of 1000 joggers and 4000 sedentary controls reported that intense joggers did not demonstrate a mortality benefit when compared to the sedentary group whereas light joggers did. It follows that there is a law of diminishing returns in relation to exercise, and the concept of a U-shaped relationship is emerging (fig. 3), although the point at which this takes effect is yet to be established.

Studies of athletes participating in prolonged exercise have demonstrated increased levels of biomarkers of cardiac damage, such as troponin [24]. The underlying mechanisms remain unclear and vary from increases in myocardial sarcolemmal permeability and integrin release, to direct myocyte necrosis. This latter process may cause individuals with a previously normal heart, who participate in repeated bouts of lifelong endurance exercise, to develop myocardial fibrosis and thus arrhythmogenic substrate (fig. 4). Research using animal models provides some support for this hypothesis. Benito et al. exercised rats for 16 weeks, which equates to 10 human years of exercise, and demonstrated eccentric left ventricular hypertrophy, diastolic dysfunction and diffuse myocardial fibrosis in the atria and right ventricle [25]. More striking, however, was the finding that ventricular fibrillation was inducible on electrophysiological stimulation in $42 \%$ of the exercised rats compared with $6 \%$ of sedentary rats.

There is further compelling evidence to suggest that long-standing participation in high-intensity sport is 


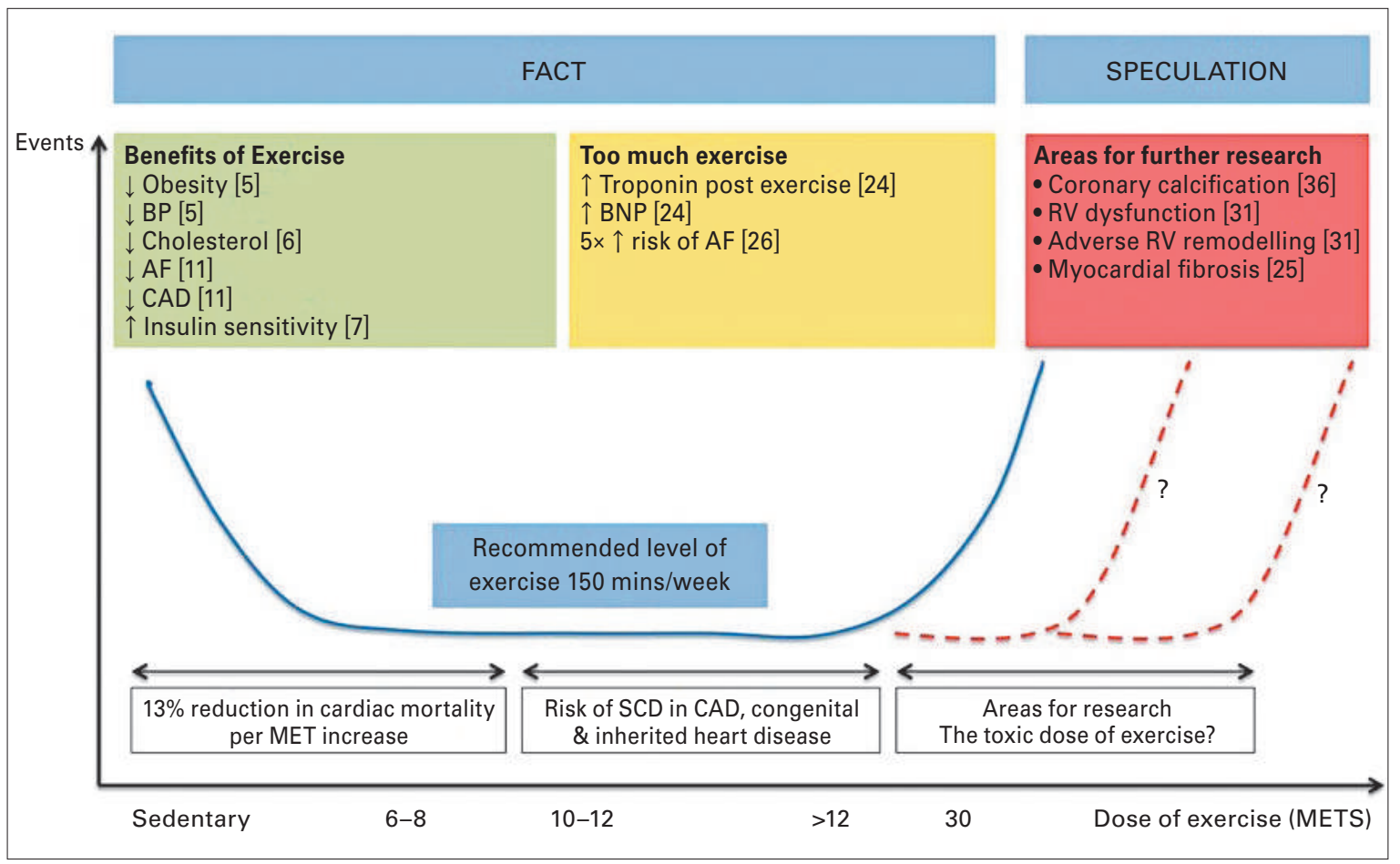

Figure 3: The $U$-shaped relationship between exercise, health and disease.

$\mathrm{AF}=$ atrial fibrillation; $\mathrm{BNP}=$ brain natriuretic peptide; $\mathrm{BP}=$ blood pressure; $\mathrm{CAD}=$ coronary artery disease; $\mathrm{MET}=\mathrm{metabolic}$ equivalent of task; $L V=$ left ventricular; $R V=$ right ventricular; $S C D=$ sudden cardiac death.

potentially detrimental for some athletes; in a metaanalysis involving 655 trained athletes, a five-fold risk of $\mathrm{AF}$ was demonstrated compared with sedentary controls [26]. Andersen et al. reported a correlation between the risk of AF and the number of completed

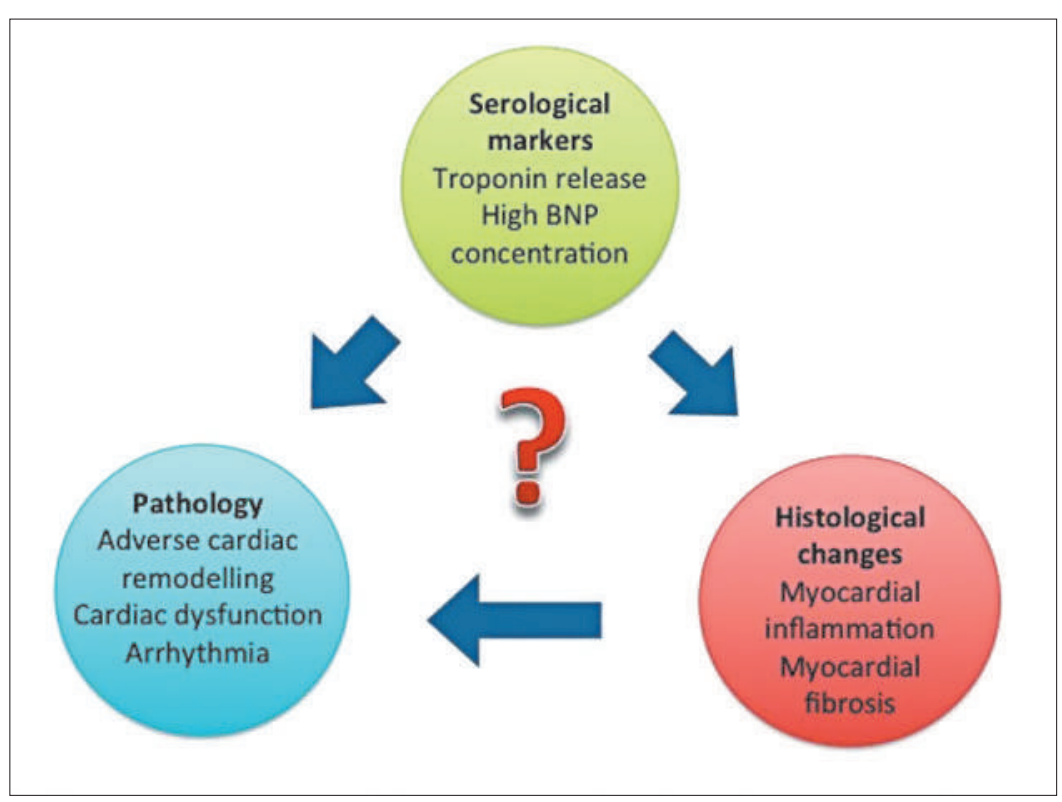

Figure 4: The possible relationship between raised serological markers, histological changes and pathology secondary to exercise.

$\mathrm{BNP}=$ brain natriuretic peptide. races and quicker times in 52000 long-distance crosscountry skiers [27]. Indeed some studies have reported an increased risk for developing $\mathrm{AF}$ in athletes who are aged 30 years and above, have a lifetime sports practice of greater than 1500 hours and partake in $>5$ hours of intense sport per week [11]. Various causal mechanisms of AF in athletes have been postulated including atrial stretch, inflammation and scarring, as well as vagally mediated shortening of the atrial refractory period [28]. Adverse atrial remodelling was demonstrated in the animal study mentioned above where rats were subjected to intensive exercise [29].

There is growing evidence that ventricular arrhythmia in a healthy athlete may be prognostically significant. In a study of 46 athletes with ventricular ectopy or nonsustained ventricular tachycardia, a high incidence of major arrhythmic events (39\%), including SCD (20\%), was observed over a 5-year follow-up period [30]. Right ventricular remodelling in the context of chronic endurance exercise has subsequently been implicated in the aetiology of such arrhythmic events [31]. La Gerche et al. used echocardiography to investigate 40 healthy athletes at baseline and after an endurance race. The study demonstrated transient right ventricular (RV) cavity enlargement and impaired RV function as well as raised cardiac troponin and B-type natriuretic peptides, which correlated with duration of exer- 
cise and degree of RV impairment. This has led to the concept of exercise-induced arrhythmogenic right ventricular cardiomyopathy (ARVC), where repeated episodes of intense endurance exercise cause adverse right ventricular remodelling and resultant susceptibility to fatal arrhythmias [32]. The dose of physical activity necessary to cause this effect is probably $>20$ hours per week for $>20$ years.

Further studies conducted in humans have assessed the role of chronic endurance exercise in myocardial fibrosis. A study of 102 males aged $\geq 50$ years, with no prior history of heart disease or diabetes and who had completed at least five marathons over the preceding three years underwent cardiac magnetic resonance imaging [33]. A three-fold greater prevalence of late gadolinium enhancement was demonstrated in the veteran athletes compared with sedentary controls ( $12 \%$ vs $3 \%, p=0.077)$.

These findings remain speculative and are mainly based on observational studies which were selective for a small minority of symptomatic athletes. In fact, the actual proportion of individuals potentially affected is tiny, when approximately 10 million athletes participate in endurance events globally every year. A study by Pellicia et al. failed to demonstrate any deterioration in cardiac function or increased risk of arrhythmia in 114 Olympic endurance athletes who had competed in two to five consecutive Olympic Games [34]. Even when a higher prevalence of ventricular arrhythmia is observed the prognostic significance remains debatable. This is highlighted in a study by Baldesberger et al. in which 134 former Swiss professional cyclists had a $15 \%$ prevalence of ventricular tachycardia when compared to $3 \%$ in age-matched golfers $(p=0.05)$. Interestingly this finding did not affect survival of the former cyclists [35].

Perhaps less dramatic than ventricular arrhythmia but still a source of significant morbidity is the higher prevalence of sinus node dysfunction and second- or third-degree block in athletes. In the study described above, $10 \%$ of the former cyclists demonstrated sinus node dysfunction compared with $2 \%$ of the golfers. A smaller study of 20 veteran athletes with a 12-year follow-up period demonstrated that $10 \%$ required permanent pacemaker implantation [36].

In the same cohort of athletes studied by Breuckmann et al., veteran marathon runners were assessed for atherosclerotic coronary artery disease using calcium scoring in a study by Mohlenkamp et al. [37]. Of these 108 male endurance athletes $36 \%$ were found to calcium scores $>100$ compared to $21 \%$ of age and Framingham risk-matched controls. Potential mechanisms included shearing forces in coronary arteries at high heart rates and inflammation causing a rise in circulating interleukins and a rise in free radical production. However, $56 \%$ of these athletes were current or former smokers, which perhaps influenced the raised calcium scores. As is the case with myocardial fibrosis, further large-scale studies are required to confirm the significance of atherosclerotic disease in the athlete.

\section{Conclusion}

The global epidemic of physical inactivity is a public health matter and exercise must be strongly encouraged. The evidence demonstrating benefit far outweighs that suggesting detriment in otherwise healthy individuals with normal hearts. To further contextualise the role of exercise, studies of athletes have repeatedly demonstrated a significantly lower all-cause mortality rate compared with sedentary controls. However, there is a higher prevalence of myocardial fibrosis and $\mathrm{AF}$ in veteran athletes. Elucidating a link between the physiological effects of high-intensity exercise and adverse clinical outcomes requires further studies addressing long-term outcomes of these rare but potentially fatal adaptations.

\section{Disclosure statement}

No financial support and no other potential conflict of interest relevant to this article was reported.

\section{References}

The full list of references is included in the online article at www.cardiovascmed.ch 